\title{
Regionalismus und Externer Einfluss: Stört die Europäische Union die Regionale Marktintegration im Südlichen Afrika?'
}

\author{
Johannes Muntschick
}

\begin{abstract}
Regionalism and External Influence: Does the EU Interfere with Regional Market Integration in Southern Africa?

Abstract: This article addresses the questions, why the rather successful process of regional market integration in the Southern African Development Community (SADC) is stalling at present and why the existence of the centennial Southern African Customs Union (SACU) is likewise at risk although the economic and political circumstances within the region have not changed much in recent times. Applying a situation-structural approach to the analysis of regional integration that takes the influence of external actors explicitly into account, the article demonstrates that the European Union has an unexpectedly interfering effect on regionalism in the South because its Economic Partnership Agreements can be made co-responsible for the failure of SADC's scheduled customs union project and an undermining of the SACU.
\end{abstract}

Keywords: Regionalism, external influence, EU, SADC, SACU

Schlagwörter: Regionalismus, externer Einfluss, EU, SADC, SACU

\section{Einleitung}

Seit Ende des Kalten Krieges lässt sich eine neue Welle von Regionalismus (Hettne u. Söderbaum 1998; Mansfield u. Milner 1999) beobachten, die sich in zahlreichen regionalen Integrationsprojekten in verschiedenen Teilen der Welt manifestiert hat. Auffällig ist, dass außerhalb der OECD-Welt und insbesondere im Süden $^{2}$ viele neue Regionalorganisationen entstanden sind, obgleich dort die strukturellen Voraussetzungen für erfolgreiche regionale Integration weitaus weniger aussichtsreich scheinen als in Gebieten mit hochindustrialisierten und wirtschaftlich eng verflochtenen Staaten. Gleichwohl haben sich außerhalb Europas

1 Der Autor bedankt sich insbesondere bei Thomas Gehring und dem Bamberger Cluster für Regionalismusforschung mit Julia Dinkel, Simon Fink, Sebastian Krapohl, Axel Obermeier und Daniel Rempe (Otto-Friedrich-Universität Bamberg). Großer Dank gilt auch Benjamin Faude und Michael Zürn (Wissenschaftszentrum Berlin für Sozialforschung), Tanja Börzel (Freie Universität Berlin), Jonathan Kaunda (Botswana Institute for Development Policy Analysis) und den beiden anonymen Gutachtern für wertvolle Kommentare und konstruktive Kritik zur Argumentation dieses Beitrags und zu früheren Arbeiten.

2 Unter dem Süden sollen jene Regionen verstanden werden, in denen hauptsächlich nicht-industrialisierte Länder mit niedrigem sozio-ökonomischem Entwicklungsstand liegen (Söderbaum u. Stålgren 2010, S. 2). 
Regionalorganisationen erfolgreich etabliert und weisen zum Teil sogar bemerkenswerte institutionelle Dynamiken auf. ${ }^{3}$

Konkurrierende Theorien wie der Neofunktionalismus (Haas 1958), der Liberale Intergouvernementalismus (Moravcsik 1998) sowie konstruktivistische (Hettne u. Söderbaum 1998) und politökonomische (Mattli 1999; Schirm 2002) Ansätze versuchen das Phänomen des Regionalismus aus verschiedenen Blickwinkeln zu erklären. Die Integrationstheorien des Mainstreams wurden jedoch vor dem Hintergrund des Erfolgsmodells des europäischen Integrationsprozesses konzipiert. Aus diesem Grund haben sie mit Blick auf die Europäische Union (EU) zwar eine hohe Erklärungskraft, greifen jedoch bei der Analyse und Erklärung von Regionalismus außerhalb Europas aufgrund ihres inhärenten Eurozentrismus zu kurz.

Regionale Integration außerhalb Europas wird bislang insbesondere von Experten aus dem Bereich der Regionalwissenschaften erforscht. Diese Abhandlungen sind zumeist empirisch sehr gehaltvoll (z. B. Mair u. Peters-Berries 2001; Vogt 2007), jedoch oft deskriptiv angelegt. Theorieorientierte Arbeiten sind selten (z. B. Acharya u. Johnston 2007; Powers u. Goertz 2011). Es existiert somit eine Forschungslücke mit Blick auf die systematische und theoriegeleitete politikwissenschaftliche Analyse von Regionalorganisationen der jüngsten Welle des Regionalismus - insbesondere in der südlichen Hemisphäre (Jetschke u. Lenz 2011; Warleigh-Lack u. Langenhove 2010). Vor diesem Hintergrund möchte der Artikel einen empirischen Beitrag zur theoriegeleiteten Analyse von Regionalismus im südlichen Afrika leisten: Er widmet sich den Fragen, warum in der Southern African Development Community (SADC) der Prozess der regionalen Marktintegration gegenwärtig ins Stocken geraten ist und warum in der Southern African Customs Union (SACU) die bereits seit langem institutionalisierte Zollunion aktuell gefährdet scheint, obwohl sich die ökonomischen Randbedingungen in der Region des südlichen Afrikas nicht verändert haben.

Die vergleichende Regionalismusforschung hat sich gegenwärtig dem Forschungsfeld ,Regionalismus außerhalb Europas' dezidiert angenommen (Jetschke u. Lenz 2011, S. 449). Neben den klassischen vertikalen Perspektiven, die Regionalismus durch endogene oder exogene Faktoren zu erklären suchen, wird in der neuen Forschung eine horizontale Perspektive eingeführt, die Diffusionsprozessen, Kulturtransfer und interregionalen Beziehungen zwischen der EU und anderen Regionalorganisationen eine wichtige Bedeutung einräumt (Börzel u. Risse 2009; Engel u. Middell 2010; Jetschke u. Lenz 2011). Diese Analyseperspektive, die externen Akteuren einen erheblichen Einfluss auf Regionalismusprozesse zugesteht, wurde von den klassischen Integrationstheorien vernachlässigt (Sbragia 2008) und bis heute nur selten thematisiert (z. B. Axline 1977; Nye 1965; Zimmerling 1991). Jüngste (Diskussions-)Beiträge der vergleichenden Regionalismusforschung zeigen allerdings, dass die horizontale Perspektive für die Analyse und zur Erklärung von Regionalismus außerhalb Europas als immer wichtiger erachtet wird (Warleigh-Lack u. Langenhove 2010).

3 Hierzu zählen z. B. die Gemeinschaft Südostasiatischer Staaten (ASEAN), die Karibische Gemeinschaft (CARICOM), der Gemeinsame Markt des Südens (Mercosur) und die Südafrikanische Entwicklungsgemeinschaft (SADC). 
Mit der Analyse von Dynamik und Status der regionalen Marktintegration in der SADC bzw. SACU soll dieser Artikel nicht nur einen empirischen Beitrag zur Debatte um den Regionalismus außerhalb Europas leisten, sondern unter Berücksichtigung der horizontalen Perspektive explizit auch den Effekt extra-regionaler Beziehungen und den Einfluss externer Akteure auf diese beiden Regionalorganisationen erklären. Die Fallbeispiele repräsentieren mit der SADC ein vielversprechendes und relativ dynamisches Beispiel von neuem Regionalismus in Afrika (Mair u. Peters-Berries 2001; Vogt 2007), wohingegen die SACU die mit Abstand älteste Regionalorganisation des Kontinents ist (McCarthy 2003). Der Fokus auf das Politikfeld der Ökonomie liegt darin begründet, dass regionale Wirtschaftskooperation den Kern von beiden Integrationsprojekten bildet.

Der Beitrag belegt, dass die EU auf Regionalorganisationen und Integrationsprozesse im südlichen Afrika nicht einen nur positiven Einfluss ausübt, wie dies häufig in der Literatur angenommen wird (z. B. Börzel u. Risse 2009), sondern für das Scheitern der geplanten SADC-Zollunion und ein drohendes Auseinanderbrechen der SACU mitverantwortlich gemacht werden kann. Der beeinträchtigende Einfluss Europas manifestiert sich vor dem Hintergrund der Economic Partnership Agreements (EPAs), die gegenwärtig zwischen der EU und Ländern im südlichen Afrika verhandelt werden. Infolgedessen vollzieht sich in beiden afrikanischen Regionalorganisationen eine interne Fragmentierung, weil verschiedene Staaten(-gruppen) eine bilaterale Kooperation mit dem externen Akteur im Rahmen der EPAs gewinnbringender werten als die vertiefte Marktintegration auf Basis ihrer eigenen Regionalorganisation.

Der Artikel gliedert sich zunächst in einen Theorieteil, in dem auf Basis von Kooperationstheorie und dem situationsstrukturellen Modell (Zürn 1992, 1993) ein theoretischer Ansatz konzipiert wird, der zentrale Annahmen zu Regionalismus und externem Einfluss formuliert und die Wirkung externer Akteure auf regionale Integrationsprojekte und deren Dynamik erklärt. Im Anschluss erfolgt im empirischen Teil die theoriegeleitete Analyse von regionaler Marktintegration im Rahmen der SADC und SACU unter besonderer Berücksichtigung ihrer Beziehungen zur EU.

\section{Regionalismus und externer Einfluss: Ein situationsstruktureller Analyseansatz}

Regionalismus kann als geplante, multilaterale und staatlich gelenkte Kooperation und Integration innerhalb eines geografisch begrenzten Raumes verstanden werden, die im Rahmen von Regionalorganisationen organisiert und institutionalisiert wird (Bach 2003, S. 22). Da Regionalismus gemäß dieser Konzeptionalisierung nicht nur auf einen Politikbereich beschränkt, sondern multidimensional ist, kann unter dem Dach einer Regionalorganisation ein ganzes Bündel von verschiedenen regionalen Kooperationsprojekten angesiedelt sein (Stein 1982, S. 316). Diese staatszentrische Herangehensweise legt den Schwerpunkt der Analyse auf Strukturen, Interdependenz und Kausalzusammenhänge auf der Makro-Ebene. Eine so praktizierte Komplexitätsreduktion ist gerade für die vergleichende Regi- 
onalismusforschung sehr sinnvoll, da so der Einfluss externer Akteure auf Regionalorganisationen fokussiert untersucht werden kann. Ferner besteht Anschlussfähigkeit an die Kooperationstheorie und die Debatte zur Entstehung von nicht-hierarchischen internationalen Regimen (Gehring u. Oberthür 1997, S. 17; Zürn 1987). Zu diesen können auch Regionalorganisationen mit einem sehr hohen Integrationsgrad - wie z. B. die EU - gezählt werden, so sie als komplexes System von ineinander verschachtelten regionalen Kooperationsprojekten und dazugehörigen Institutionen interpretiert werden (Gehring 1994, S. 216; Moravcsik 1998, S. 15).

\subsection{Situationsstruktur und Regionale Kooperation}

Der Bedarf an regionaler Kooperation und Integration kann auf die Struktur des internationalen Systems und die ihm inhärenten problematischen Situationen (Zürn 1993) zurückgeführt werden, die durch komplexe Interdependenz zwischen den Staaten entstehen (Hurrell 1995; Keohane 1984, S. 15). Vor dem Hintergrund einer regionalen Kooperationsproblematik können internationale Institutionen rational nutzenmaximierenden Akteuren Anreize bieten, ihr unilaterales Handeln in kooperatives Verhalten zu ändern, wenn die zu erwartenden Kooperationsgewinne den Nutzen des unkoordinierten Status quo übertreffen (Axelrod u. Keohane 1993). Effektive Regionalorganisationen können dann durch ihre regulative und ,zivilisierende' Wirkung zu Wohlfahrt, Stabilität und Frieden einer Region und ihrer Staaten beitragen (Zürn 1987, S. 6, 36, 44-45).

Der situationsstrukturelle Ansatz modelliert problematische Situationen in den internationalen Beziehungen anhand von ,Spielen' (Zürn 1992, 1993), die in ihrer Typisierung an die Spieltheorie angelehnt sind (Axelrod 1987). Sie stellen die notwendige Bedingung dar, die bei Staaten den Bedarf für institutionalisierte regionale Kooperation generiert. Der situationsstrukturelle Ansatz unterscheidet drei Idealtypen von problematischen Situationen, die aufgrund der zugrunde liegenden Struktur einen unterschiedlichen Grad an kooperativer Lösbarkeit implizieren: So ist diese am einfachsten in Situationen zu erreichen, die Koordinationsspielen ähneln, wohingegen jene Spiele des Gefangenendilemma-Typs deutlich schwieriger $\mathrm{zu}$ lösen sind und Kooperation in sogenannten ,Rambo'-Spielen fast ganz unmöglich ist (Zürn 1992). Prinzipiell hängt es somit von der Struktur der zugrunde liegenden Situation $a b$, ob und wie internationale Kooperation realisiert werden kann und wie stark der Bedarf für regulative Institutionen ist (Zürn 1993, S. 6970). Die Entstehung von Regionalismus, insbesondere mit Blick auf das institutionelle Design und Regelwerk der Kooperationsprojekte, stellt dann die abhängige Variable dar, die es zu erklären gilt.

Je nach Situationsstruktur können intervenierende Kontextvariablen diesen Prozess beeinflussen und so die Chance für erfolgreiche internationale Kooperation maßgeblich verbessern oder verringern. Während der Einfluss von Kontextvariablen bei problematischen Situationen, die an Koordinationsspiele erinnern, aufgrund der Salienz von Lösungen prinzipiell eher gering ist, so ist er bei Gefangenendilemmata oder Rambo-Konstellationen von substanzieller Bedeutung. Unter allen denkbaren Kontextvariablen wird die größte Relevanz dem As- 
pekt der Machtverteilung zugeschrieben (Zürn 1993, S. 70-71), die vom Charakter der politikfeldspezifischen Interdependenzbeziehung zwischen den beteiligten Akteuren abgeleitet werden kann (Keohane u. Nye 2001). Diesem Faktor wird im Rahmen der situationsstrukturellen Analyse von Regionalismus daher die zentrale Beachtung beigemessen.

Zwar haben rational nutzenmaximierende Akteure das gemeinsame Interesse, aus kollektivem Handeln absolute Kooperationsgewinne zu erzielen, doch sind die individuellen Präferenzen mit Blick auf die Verteilung der Gewinne und anfallenden Kosten, d. h. der relativen Gewinne, insbesondere bei Mixed-Motive-Spielen nicht kongruent. Um die individuell besten Ergebnisse zu erlangen, treten die Akteure zur Lösung dieser Second-Order-Probleme in Verhandlungen über Konditionen und institutionelle Ausgestaltung der Kooperationsvereinbarung (Zangl 1994, S. 284-287). Gemäß intergouvernementaler Verhandlungstheorie deutet asymmetrische Interdependenz auf eine relative Machtposition bei jenen Akteuren hin, die auf Basis des Vorhandenseins von attraktiven, unilateralen PolicyAlternativen und Exit-Optionen glaubhaft mit dem Abbruch von Verhandlungen drohen können (Hirschmann 1945, S. 16; Keohane u. Nye 2001, S. 9-10, 268270).

Jene Akteure, die eine zentrale Position im Beziehungsgeflecht einnehmen und von denen andere abhängig sind, konstituieren daher aus strukturellen Gründen die Eckpfeiler für den Erfolg eines Kooperationsprojektes und können ihr Engagement von Kompromissbereitschaft und Konzessionen der schwächeren Partner abhängig machen (Gehring 1994, S. 216; Moravcsik 1998, S. 64-65). Eine Kooperationsvereinbarung und ihr institutionelles Design spiegeln daher nicht nur die Konstellation der jeweiligen Präferenzen im Kooperationsfeld wider, sondern gleichzeitig auch die Interdependenzbeziehung zwischen den Akteuren und ihre daraus resultierende relative Machtposition. Der Faktor Macht verleiht somit regionalen Großmächten eine Schlüsselrolle im Hinblick auf die Entstehung und Dynamik von Regionalismus (Keohane 1988, S. 387; Zürn 1993, S. 70).

\subsection{Regionale Kooperation und externer Einfluss: Die Ambivalenz von asymmetrischer extra-regionaler Interdependenz}

Ein auf Kooperationstheorie basierender situationsstruktureller Ansatz ist frei von eurozentrischen Prämissen und kann somit eine globale Gültigkeit und Erklärungskraft beanspruchen. Demzufolge könnte man zunächst annehmen, dass Regionalismus im Süden grundsätzlich derselben Logik folgt wie in anderen Teilen der Welt. Obgleich dies ceteris paribus prinzipiell zutreffend ist, vernachlässigt diese Annahme eine zentrale strukturelle Rahmenbedingung, der insbesondere Regionen im Süden ausgesetzt sind. Nicht zuletzt aufgrund der kolonialen Vergangenheit weisen dort die Länder häufig eine starke asymmetrische extra-regionale Interdependenz zu mächtigen globalen Akteuren auf, die im Bereich der Wirtschaft besonders akzentuiert ist (Young 1969, S. 727).

Um die horizontale Ebene und den externen Einfluss für die Erklärung der Entstehung und Dynamik von Regionalismus angemessen zu berücksichtigen, soll 
daher das Strukturmerkmal der extra-regionalen Interdependenz als zusätzliche Kontextvariable konzeptualisiert werden, die es bei der Analyse zu prüfen gilt. So kann asymmetrische extra-regionale Interdependenz der regionalen Akteure die zugrunde liegende Struktur von genuin regionalen Kooperationsproblemen transformieren und dadurch eine Lösbarkeit potenziell erschweren oder begünstigen. Dies gilt sowohl für die Ebene der Problemstruktur als auch für jene der SecondOrder-Problematik und Lösungsfindung (Axline 1977; Burns u. Buckley 1974).

Mit Blick auf die Problemstruktur kann sich dies darin manifestieren, dass sich die klassische Situation eines Gefangenendilemmas in Richtung eines kooperationsaversen Rambo-Spiels verändert, bei dem auf regionaler Ebene jene Akteure zu unkooperativen ,Rambos' werden, die auf Basis von extra-regionalen Beziehungen über attraktivere (Kooperations-)Optionen außerhalb der eigenen Region verfügen (Rohlfing 2009). Andererseits kann Regionalismus jedoch auch von außen gefördert werden, wenn externe Akteure die Lösung von genuin regionalen Kooperationsproblemen durch eine Erhöhung der dort zu erwartenden Kooperationsgewinne attraktiver machen. Das kann beispielsweise durch das Setzen von Kooperationsanreizen oder das Senken der Institutionalisierungskosten geschehen (Nye 1965). Mit Blick auf die zugrunde liegende Problemstruktur würde sich dann eine genuine Rambo-Situation zu einem Gefangenendilemma oder gar zu einem noch kooperationsgeneigteren Koordinationsspiel mit Verteilungskonflikt transformieren (Axline 1977, S. 100-102; Axline 1994, S. 25-26; Burns u. Buckley 1974).

In zwischenstaatlichen Verhandlungen auf regionaler Ebene kann extra-regionale Interdependenz mit Blick auf das zu vereinbarende Kooperationsergebnis ebenfalls eine potenzielle Wirkung entfalten. Starke Beziehungen zu externen Akteuren können die Verhandlungsmacht eines Staates im regionalen Verbund sogar bei eindeutig regionalorientierten Themen relativ vergrößern, da sie ein Vorhandensein von alternativen Policy-Strategien und plausiblen Exit-Optionen, die außerhalb der Reichweite der Region liegen, implizieren. Der Handlungsspielraum bei diesen Staaten wird somit durch eine relativ geringere Abhängigkeit von den intra-regionalen Kooperationsproblemen und den damit verbundenen Verhandlungsergebnissen und Institutionen signifikant erweitert (Sebenius 1983). Eine auf diese Weise gestärkte Verhandlungsmacht ist jedoch vergleichsweise instabil, da sie durch das Verhältnis zu externen Akteuren determiniert ist. Sie schwindet, sobald die extra-regionalen Policy-Alternativen und Exit-Optionen von dritter Seite einseitig verschlossen werden. Externe Akteure können die Verhandlungsposition und -strategie von regionalen Akteuren auf regionaler Ebene durch positive oder negative Anreize auch direkt beeinflussen, so sie sich denn aufgrund eines asymmetrischen extra-regionalen Interdependenzverhältnisses in der Position der Stärke befinden (Axline 1994, S. 23-26).

Der so skizzierte externe Einfluss auf regionale Problemstrukturen und den Handlungsspielraum von regionalen Akteuren kann somit prinzipiell ambivalente Auswirkungen auf die Gesamteffektivität von einzelnen Kooperationsprojekten oder ganzen Regionalorganisationen haben. 


\subsection{Synopse und zentrale Annahmen}

Entstehung und Dynamiken von Regionalismus folgen prinzipiell weltweit derselben Logik und hängen primär von der Struktur des zugrunde liegenden Kooperationsproblems ab: In Situationen, die einem Koordinationsspiel oder Gefangenendilemma ähneln, ist erfolgreiche regionale Kooperation wahrscheinlich, wohingegen dies bei Rambo-Situationen nahezu unmöglich ist (Zürn 1992, 1993). Staaten, die sich in einer regionalen Machtposition befinden, können dabei Gestalt und Dynamik von Regionalismus maßgeblich positiv oder negativ beeinflussen (Keohane u. Nye 2001).

Weisen Staaten einer Region starke asymmetrische extra-regionale Interdependenz auf, können die in Beziehung stehenden externen Akteure die Evolution von regionaler Kooperation dort (in-)direkt beeinflussen: Regionalismus wird erschwert, wenn sich aufgrund von erfolgversprechenderen extra-regionalen Beziehungen die genuine Struktur des regionalen Kooperationsproblems zu einer Rambo-Situation transformiert. Regionalismus wird dagegen begünstigt und gefördert, wenn Externe an der Generierung des Klubgutes mitwirken oder es gar maßgeblich bereitstellen, da sich so die genuine problematische Situation in Richtung eines Koordinationsspiels (mit Verteilungskonflikt) wandelt. Zugleich können starke Beziehungen zu Externen, je nach Ausprägung und Grad der Asymmetrie, die Verhandlungsmacht von Akteuren auf der regionalen Ebene erweitern oder einschränken. Obgleich der Einfluss von außen somit prinzipiell ambivalent sein kann, ist insbesondere von negativen Effekten auszugehen, da den externen Akteuren individuell nutzenmaximierendes Handeln unterstellt werden muss. Unter Berücksichtigung dieser Annahmen sollen im Rahmen einer Plausibilitätsprobe (Eckstein 1975, S. 108-113) nun Dynamik und Status von regionaler Marktintegration in der SADC und SACU analysiert und erklärt werden.

\section{Regionalismus im Schatten der EU: Der negative Effekt der Economic Partnership Agreements auf regionale Marktintegration in der SADC und SACU}

Das südliche Afrika weist die Besonderheit auf, dass hier mit der SADC und SACU eine relativ neue und eine sehr alte Regionalorganisation parallel existieren. Die SADC wurde 1992 als Nachfolgeorganisationen der Southern African Development Co-ordination Conference (SADCC) gegründet und zählt heute 15 Mitgliedstaaten ${ }^{4}$. In Afrika gilt sie als aussichtsreiche und dynamische Regionalorganisation, die sich die Entwicklung der Region und ihrer Mitgliedstaaten in einer Reihe von Politikfeldern auf die Fahnen geschrieben hat. Seit Mitte der 1990er-Jahre legt die SADC den Schwerpunkt auf regionale Wirtschaftsintegration (Oosthuizen 2006; Vogt 2007). Der Regional Indicative Strategic Develop-

4 Die Mitgliedstaaten der SADC sind Angola, Botsuana, die DR Kongo, Lesotho, Madagaskar, Malawi, Mauritius, Mosambik, Namibia, Sambia, die Seychellen, Simbabwe, Südafrika, Swasiland und Tansania. Die Mitgliedschaft von Madagaskar ist gegenwärtig aufgrund des jüngsten Putsches ausgesetzt. 
ment Plan (RISDP), der im Jahr 2004 von der SADC verabschiedet wurde, hat die Schaffung der SADC-Zollunion für das Jahr 2010 terminiert (SADC 2004a), nachdem die Freihandelszone bereits im August 2008 erfolgreich etabliert wurde.

Die SACU wurde auf maßgebliches Betreiben Südafrikas ${ }^{5}$ im Jahr 1910 gegründet und umfasst fünf Mitgliedstaaten ${ }^{6}$. Sie gilt als älteste funktionierende Zollunion der Welt, hat einen gemeinsamen Außenzoll und gewährleistet im Inneren den freien Verkehr von Waren und vielen Dienstleistungen. Die Zolleinnahmen fließen in einen gemeinsamen Pool und werden jährlich auf Basis einer Formel an die Mitgliedstaaten verteilt. In den Jahren 1969 und 2002 gab es jeweils institutionelle Reformen, die den BLNS-Staaten ${ }^{7}$ einen größeren Anteil an den Zolleinnahmen und mehr Entscheidungsbefugnisse in der Organisation zusprachen (Bertelsmann-Scott 2010).

\subsection{Monozentrische intra-regionale ökonomische Interdependenz: Südafrika als regionale Wirtschaftsmacht}

Mit Ausnahme von Botsuana, Namibia, Mauritius, den Seychellen und der Republik Südafrika (RSA) zählen alle Staaten im südlichen Afrika zu den Least Developed Countries (LDC) ${ }^{8}$ oder gehören zu den Kategorien von Ländern mit geringem bzw. mittlerem Einkommen im unteren Bereich. ${ }^{9}$ Die intra-regionale Wirtschaftsverflechtung ist im Vergleich zu anderen Weltregionen relativ schwach ausgeprägt. In der SADC belief sich der formale intra-regionale Handel zwischen 2000 und 2007 lediglich auf ca. 13-15 Prozent der Importe bzw. 15-21 Prozent der Exporte (Krapohl u. Muntschick 2009, S. 19) und in der SACU im selben Zeitraum sogar nur auf rund 6-10 Prozent des Gesamthandels. ${ }^{10}$

Gleichwohl gibt es im südlichen Afrika durchaus einen Bedarf an Handelsliberalisierung und regionaler Marktintegration: Während die wirtschaftlich weniger entwickelten ${ }^{11}$ Länder der Region hauptsächlich Rohstoffe und (verarbeitete) Nahrungsmittel exportieren, ist gerade die Wirtschaft Südafrikas vergleichsweise diversifiziert und zählt neben Rohstoffen auch kapitalintensive Industrieprodukte zum Spektrum ihrer Exportgüter. Der zu Zeiten der Apartheid noch hoch regulierte südafrikanische Markt bietet für seine Nachbarn Absatzmöglichkeiten für Rohstoffe und geringfügig verarbeitete Produkte, wohingegen für Südafrika die Region eine wichtige Bedeutung als Markt für jene Industriegüter hat, die global

5 Damals noch die Südafrikanische Union, die erst 1961 zur Republik Südafrika wurde.

6 Die Mitgliedstaaten der SACU sind Botsuana, Lesotho, Namibia, Südafrika und Swasiland.

7 Botsuana, Lesotho, Namibia und Swasiland.

8 Kategorisierung der Vereinten Nationen. http://www.unohrlls.org/en/ldc/25/. 04.09.2012.

9 Kategorisierung der Weltbank. http://siteresources.worldbank.org/DATASTATISTICS/Resources/ CLASS.XLS. 04.09.2012.

10 RIKS - Indicators and Statistics of Regional Agreements: http://www.cris.unu.edu/riks/web/data/ show. 10.07.2012.

11 Die Bezeichnung, entwickelte' und ,weniger entwickelte‘ Länder wird im Folgenden in Anlehnung an die sehr gängigen Kategorisierungen der Vereinten Nationen und Weltbank verwendet, obgleich deren Terminologie und Differenzierungen auch nicht völlig unumstritten sind. Der Autor bedankt sich in diesem Zusammenhang bei Gutachter 2 und Alexander Brand (Johannes Gutenberg-Universität Mainz) für ihre hilfreichen Hinweise. 
nicht konkurrenzfähig sind (Draper et al. 2006, S. 73-81). Durch regionale Handelsliberalisierung könnte ferner ein Großteil des informellen Handels, der in der Region insbesondere mit Agrarprodukten betrieben wird, in formelle Bahnen gelenkt werden (Asche 2008, S. 102; Sandrey 2011, S. 74-77).

In der Region offenbart sich eine beachtliche asymmetrische ökonomische Interdependenz, die einem Speichennetz ähnelt, dessen Zentrum Südafrika bildet: Die Kaprepublik ist seit der Kolonialzeit der unangefochtene Wirtschaftsgigant im südlichen Afrika und trägt rund 70 Prozent zum Bruttoinlandsprodukt und ca. 65 Prozent zum Gesamthandelsvolumen der Region bei. Seit Jahrzehnten weist Südafrika dabei eine sehr positive Handelsbilanz auf, zumal Pretoria der wichtigste Handelspartner für gut die Hälfte der Länder in der Region ist (Oosthuizen 2006, S. 255-261). Die Asymmetrie dieser Wirtschaftsbeziehungen kann durch den Charakter der intra-regionalen Handelsströme belegt werden: Rund die Hälfte der SADC-Staaten handelt mit Blick auf das Gesamthandelsvolumen mehr mit Partnern in der eigenen Region als mit externen Akteuren, wobei der regionale Markt insbesondere für Swasiland (78,5 Prozent), Simbabwe (66,5 Prozent) Namibia (38,0 Prozent), Malawi (35,7 Prozent), Sambia (23,2 Prozent) und Mosambik (22,5 Prozent) eine zentrale oder gar die wichtigste Exportdestination darstellt (Anteil der Exporte in die SADC an den Gesamtexporten für 2007). Das Gros dieses intra-regionalen Handelsvolumens beruht dabei auf dem Warenverkehr zwischen Südafrika und den übrigen SADC-Staaten, da letztere untereinander vergleichsweise wenig Handel betreiben. Für die RSA ist die Abhängigkeit vom regionalen Markt dagegen vergleichsweise gering, da nur 10,1 Prozent der südafrikanischen Gesamtexporte ihren Weg dorthin finden. ${ }^{12}$ Diese monozentrische Struktur des intra-regionalen Handels kann mit noch stärkerer Akzentuierung auch in der SACU beobachtet werden (SACU 2009).

Ein Blick auf die Ströme der intra-regionalen ausländischen Direktinvestitionen erhärtet den Eindruck von asymmetrischer ökonomischer Interdependenz in der Region: Die Mehrzahl der Länder ist in hohem Maße von Südafrika abhängig, das an den gesamten ausländischen Direktinvestitionen für die übrige Region den größten Anteil trägt (Draper et al. 2010). Für sieben Staaten der Region war Südafrika zwischen 1994 und 2003 der bedeutendste ausländische Investor, wobei der südafrikanische Anteil an den gesamten ausländischen Direktinvestitionen insbesondere in Lesotho (86 Prozent), Malawi (80 Prozent), Swasiland (71 Prozent), DR Kongo (71 Prozent) und Botsuana (58 Prozent) sehr bedeutsam ist (Grobbelaar 2004, S. 93-95). Pretoria stellt auch in jüngster Zeit für das übrige südliche Afrika eine wichtigere Quelle von Investitionen dar als globale Akteure wie die EU, USA, Japan oder China. ${ }^{13}$

Die BLNS-Staaten stehen im Vergleich zu den übrigen Ländern der Region zusätzlich noch in einem weiteren, ungleich stärkeren wirtschaftlichen Abhängig-

12 Daten beruhen auf der SADC-Trade Database (http://data.sadctrade.org/st) und wurden mit der World Integrated Trade Solutions (WITS) auf Basis von UN COMTRADE ergänzt. https://wits. worldbank.org/WITS/. 10.07.2012.

13 Interview mit Mzukisi Qobo (Head of Emerging Powers Programme am South African Institute of International Affairs) im August 2010. 
keitsverhältnis zu Südafrika. Durch ihre Mitgliedschaft in der SACU, die ökonomisch und institutionell seit ihrer Gründung von Pretoria dominiert wird, stehen ihnen jedes Jahr Anteile der gemeinsamen Zolleinnahmen zu (Bertelsmann-Scott 2010). Diese steuern in Swasiland (63 Prozent), Lesotho (55 Prozent), Namibia (40 Prozent) und Botsuana (20 Prozent) bis heute einen erheblichen Anteil zum nationalen Gesamtbudget bei. Während somit die BLNS-Länder mit Blick auf ihre Staatshaushalte seit ihrer Unabhängigkeit in hohem Maße von der südafrikanisch dominierten SACU abhängig sind, gilt dies für Pretoria nicht, denn die Zolleinnahmen tragen dort lediglich knapp 4 Prozent zum nationalen Budget bei (SACU 2009).

Aufgrund dieser monozentrischen intra-regionalen Asymmetrie könnte man erwarten, dass Südafrika als regionale Großmacht die Gründung und institutionelle Ausgestaltung der geplanten SADC-Zollunion maßgeblich beeinflussen und vorantreiben kann, wie dies bereits bei der im Jahr 2008 vollendeten SADC-Freihandelszone der Fall war. Auch mit Blick auf die Funktionalität und Zukunft der SACU befindet sich Südafrika deswegen in der Schlüsselposition. Die Perspektiven für regionale Marktintegration in der SADC und SACU scheinen somit auf den ersten Blick günstig zu sein, solange dieser Prozess von Südafrika, dem regionalen Hegemon, positiv beeinflusst wird.

\subsection{Stark asymmetrische Nord-Süd-Wirtschaftsbeziehungen: Die EU als wichtigster externer Handelspartner für das südliche Afrika}

Im Gegensatz zu breiten Teilen der nördlichen Hemisphäre konzentriert sich die wirtschaftliche Verflechtung der Regionalorganisationen und ihrer Mitgliedsländer gerade im südlichen Afrika jedoch nicht nur auf die eigene Region. Dieser strukturelle Unterschied wird daran deutlich, dass mit der EU ein externer Akteur der größte Handelspartner und Exportmarkt für die SADC und SACU in ihrer Gesamtheit, für Südafrika als deren wirtschaftliches Zentrum und für rund die Hälfte der übrigen Staaten der Region, ist. Im Vorfeld der geplanten SADC-Zollunion betrug der Handel mit der EU bei SADC und SACU rund 29 Prozent bzw. 31 Prozent ihres Gesamthandelsvolumens und jeweils gut 33 Prozent ihrer Gesamtexporte (2008). ${ }^{14}$ Disaggregiert man diese Daten, so ist die EU abgesehen von Südafrika (33,2 Prozent) insbesondere für Mauritius (70,2 Prozent), Botsuana (67,7 Prozent), Madagaskar (62,9 Prozent), die DR Kongo (54,3 Prozent), die Seychellen (53,6 Prozent), Namibia (44,7 Prozent) und Malawi (39,0 Prozent) eine zentrale bzw. die wichtigste Exportdestination (Anteil an den Gesamtexporten für 2007). ${ }^{15}$ Sie stellt damit für gut die Hälfte der SADC-Staaten einen wichtigeren Handelspartner und insbesondere Exportmarkt dar als die eigene Region. Für die SACU gilt dies nicht in gleichem Maße, denn abgesehen von Südafrika

14 Daten mit der World Integrated Trade Solutions (WITS) auf Basis von UN COMTRADE erhoben. https://wits.worldbank.org/WITS/. 10.07.2012.

15 Daten beruhen auf der SADC-Trade Database (http://data.sadctrade.org/st) und wurden mit der World Integrated Trade Solutions (WITS) auf Basis von UN COMTRADE ergänzt. https://wits. worldbank.org/WITS/. 10.07.2012. 
handelt nur Botsuana mehr mit der EU als mit Partnern der eigenen Regionalorganisation. ${ }^{16}$

Während somit viele SADC- und SACU-Länder eine starke asymmetrische extra-regionale ökonomische Interdependenz aufweisen und sich in ihrer strukturellen Abhängigkeit vom europäischen Markt sehr ähnlich sind, unterscheiden sie sich doch hinsichtlich der Produktpalette ihrer Exporte: Rohstoffe und Güter aus dem Primärbereich dominieren zwar generell die Exporte in die EU, aber die Aggregatdaten verschleiern die Varianz zwischen den Ausfuhren der individuellen Länder, die sich z. B. auf Erdöl (Angola), Fisch und Rindfleisch (Namibia, Mosambik bzw. Botsuana), Zucker (Swasiland), Maschinen und Industrieprodukte (Südafrika) oder verschiedene (verarbeitete) Nahrungs- und Genussmittel (Lesotho, Malawi, Simbabwe, Südafrika) konzentrieren. ${ }^{17}$ Bei den Staaten des südlichen Afrikas ist die jeweilige Zusammensetzung der Produktpalette der für den Export in die EU bestimmten Güter somit durchaus heterogen, sodass auch der Grad ihrer Abhängigkeit vom europäischen Partner nicht gänzlich gleichartig ist (Keck u. Piermartini 2008, S. 92-94).

Im Gegensatz zur großen Bedeutung der EU als Handelspartner und Exportziel für viele Volkswirtschaften des südlichen Afrikas sind die Märkte der SACU und SADC für die EU nur von marginalem Belang, da nur knapp 2 Prozent bzw. gut 2,5 Prozent der Gesamtexporte gegenwärtig dorthin fließen. ${ }^{18}$ Einzig Südafrika stellt als Schwellenland, regionale Wirtschaftsmacht und Handelsdrehkreuz im südlichen Afrika diesbezüglich eine Ausnahme dar. Die asymmetrische ökonomische Interdependenz zwischen Nord und Süd spiegelt dabei noch rudimentär jenes Handelsverhältnis wider, wie es lange zwischen europäischen Kolonialmächten und ihren abhängigen Gebieten in Übersee bestand (Olivier 2006).

Dieser Eindruck verstärkt sich mit Blick auf den Fluss der extra-regionalen ausländischen Direktinvestitionen: Diese sind höchst asymmetrisch und unidirektional, denn sowohl die SADC als auch die SACU beziehen in ihrer Gesamtheit, in absoluten Zahlen, die meisten ausländischen Direktinvestitionen von externen Akteuren, insbesondere von der EU. Dabei erhält Südafrika als wirtschaftliches Zentrum beider Regionalorganisationen den mit Abstand größten Teil aller von außerhalb der Region stammenden ausländischen Investitionen (Sandrey 2012, S. 190-191). Im Gegensatz dazu sind die aus dem südlichen Afrika stammenden Direktinvestitionen für die EU vernachlässigbar, was die Asymmetrie der ökonomischen Beziehungen zwischen den beiden Regionalorganisationen weiter erhärtet (Goldstein 2004, S. 45-46).

Vor dem Hintergrund der intensiven (Handels-)Beziehungen zur EU, die Regionalismus und wirtschaftliche Blockbildung im Süden durch eine Vielzahl von Mechanismen und Programmen ostentativ und wohlwollend fördert (Pietrangeli 2009), ist gerade die SADC in den Genuss von erheblichen Mitteln der Entwick-

16 Eine anschauliche Darstellung von intra- und extra-regionalen Handelsströmen der SADC-Staaten in Form von Netzwerkgrafiken findet sich bei Krapohl und Fink (2009).

17 Daten mit der World Integrated Trade Solutions (WITS) auf Basis von UN COMTRADE erhoben. https://wits.worldbank.org/WITS/. 10.07.2012.

18 Daten beruhen auf Eurostat.

http://epp.eurostat.ec.europa.eu/portal/page/portal/international_trade/data/database. 06.07.2012. 
lungszusammenarbeit gekommen. Die EU unterstützt Bemühungen der SADC zur regionalen Integration explizit und stellt der Regionalorganisation als deren größter Geber im Rahmen des zehnten Europäischen Entwicklungsfonds (2008-2013) gegenwärtig 116 Millionen Euro zur Verfügung (European Community - Southern African Region 2008, S. IV). Ferner trägt die EU auch traditionell den bei weitem größten Anteil zum Gesamtbudget der SADC bei, das im Haushaltsjahr 2011/12 zu gut 72 Prozent von externen Akteuren finanziert wurde (SADC 2011). Die SACU ist dagegen seit jeher nicht von externen Fördergeldern abhängig. Im Jahr 2010 trugen finanzielle Zuwendungen der EU nur 5,5 Prozent zum Budget der Organisation bei und sanken bis 2011 auf Null (SACU 2011, S. 47).

Zusammenfassend lässt sich feststellen, dass wegen der starken asymmetrischen extra-regionalen ökonomischen Beziehungen einiger SADC-/SACU-Staaten zur EU die Gestaltung und Dynamik von Marktintegration nicht nur isoliert als regionales Vorhaben im gänzlich regionalen Rahmen betrachtet werden kann. Dieses besondere Strukturmerkmal unterstreicht die Bedeutung der horizontalen Ebene für den Regionalismus im südlichen Afrika. Leitet man nun von den intraund extra-regionalen ökonomischen Asymmetrien den Faktor Macht als intervenierende Kontextvariable ab, so kommt man zu dem Schluss, dass neben dem regionalen Hegemon Südafrika insbesondere auch die EU als zentraler externer Akteur die regionalen Marktintegrationsprojekte in der SADC und SACU beeinflussen kann.

\subsection{Gestörte Marktintegration in der SADC: Das absehbare Scheitern der geplanten Zollunion}

Die Schaffung der SADC-Zollunion, die laut RISDP bereits für das Jahr 2010 geplant war, ist ein essenzieller Eckpunkt in der Agenda zur regionalen Marktintegration (SADC 2004a). Seit Jahren wird in Dokumenten der SADC und von SADC-Offiziellen ${ }^{19}$ der wirtschaftliche und politische Nutzen dieser gemeinsamen Zollunion betont (SADC 2004b, S. 303; SADC 2006a, Artikel 7; SADC 2008, S. 10). Auch Regierungsvertreter ${ }^{20}$ der Mitgliedstaaten bekräftigten regelmäßig ihr ,Commitment' zur vertieften Marktintegration und für das gesetzte Ziel. Das SADC-Sekretariat stellte schließlich im Jahr 2006 einen Vertragsentwurf mit elaboriertem Regelwerk für die geplante Zollunion vor, was für den erwarteten Mehrwert dieses Projektes aus Sicht der regionalen Akteure spricht (SADC 2006b). Wenn man die regionale Situation isoliert betrachtet und externe Akteure zunächst nicht berücksichtigt, könnte man die zugrunde liegende Kooperationsproblematik vor diesem Hintergrund und angesichts des vorhandenen Handelspotenzials innerhalb der SADC als Gefangenendilemma interpretieren. Institutio-

19 Interview mit Juma Kaniki (Senior Programme Manager Microeconomic Monitoring and Performance Surveillance am SADC Directorate of Trade, Industry, Finance and Investment) im Januar 2008.

20 Thabo Mbeki, damaliger Präsident Südafrikas. Rede vom 14.11.2006 beim SADC Parliamentary Forum. http://www.dfa.gov.za/docs/speeches/2006/mbek1114.htm. 10.09.2012.

Pakalitha Mosisili, damaliger Premierminister Lesothos. Interview mit dem Nam News Network am 23.10.2006. http://www.namnewsnetwork.org/v3/read.php?id=MTMxODY=. 10.09.2012. 
nalisierte Handelsliberalisierung im Rahmen einer SADC-Zollunion würde unter dieser Annahme alle beteiligten Staaten besser stellen, selbst wenn nur eine mäßige intra-regionale ökonomische Interdependenz vorhanden ist.

Im Fall der SADC-Zollunion kann jedoch ein negativer Einfluss der EU auf ein zentrales Kooperationsprojekt einer Regionalorganisation des Südens konstatiert werden. Um dem Regelwerk der Welthandelsorganisation (WTO) zu entsprechen, musste die EU ihre Handelsbeziehungen zur Gruppe der afrikanischen, karibischen und pazifischen Staaten (AKP-Staaten) auf eine neue vertragliche Grundlage stellen, nachdem die nicht-reziproken Handelsvereinbarungen der Lomé-IVKonvention zum Jahr 2000 ausgelaufen waren. Das im Juni gleichen Jahres unterzeichnete Cotonou-Abkommen (Europäische Gemeinschaft 2000 [CotonouAbkommen]) bildet den Rahmen für die Neuregelung dieser Handelsbeziehungen und verlangt von EU und AKP-Staaten den präferenziellen Marktzugang auf Basis von Reziprozität. Als flankierende Maßnahmen erlaubt es die Aushandlung von EPAs, um mögliche negative Folgen der Handelsliberalisierung auf die wirtschaftlich schwächeren Partner abzufedern (Söderbaum 2007, S. 196-198). Die EPAs sind laut EU als Instrumente für die Entwicklung der wirtschaftlich schwächeren AKP-Staaten konzipiert und sollen explizit bestehende Regionalorganisationen und wirtschaftliche Integrationsprojekte in diesen Räumen fördern (Europäische Kommission 2002).

Von den 15 Mitgliedern der SADC sind all jene von dieser Neuordnung ihrer Handelsbeziehungen zur EU betroffen, die nicht zur Kategorie der am wenigsten entwickelten Länder zählen und somit nicht in den Genuss des zoll- und kontingentfreien Marktzugangs auf Grundlage der Everything-But-Arms (EBA) Initiative kommen (Europäische Union 2005 [Everything-But-Arms Initiative]). Sie müssen daher mit der EU ein EPA vereinbaren, wenn sie ihren präferenziellen Zugang zum europäischen Exportmarkt wahren wollen (Keck u. Piermartini 2008).

Da die SADC im Gegensatz zur EU bislang lediglich das Stadium einer Freihandelszone erreicht hat, gibt es keinen gemeinsamen Außenzoll und keine für alle Mitglieder verbindliche Handelspolitik gegenüber externen Akteuren. Individuell divergierende Handelsabkommen von einzelnen Staaten mit Dritten stellen für ein solches, eher schwach institutionalisiertes Kooperationsprojekt daher auch kein Problem dar. ${ }^{21}$ Um die geplante Zollunion vollenden zu können (Viner 1950), müssten sich jedoch alle SADC-Staaten in den laufenden EPA-Verhandlungen zwingend auf einen gemeinsamen Nenner einigen und der EU ein einziges, gemeinsames Angebot unterbreiten.

Ein koordiniertes, gemeinsames Auftreten aller Mitglieder der SADC, als einheitlicher Akteur, findet jedoch nicht statt. Dies liegt zum einen daran, dass dem SADC-Sekretariat das Mandat und die Kapazität für direkte Verhandlungen mit der EU im Interesse der Gesamtorganisation fehlen, sodass es sich auf die Bereit-

21 So ist die SADC-Freihandelszone mit dritten, extra-regionalen Handelsregimen in Form von Freihandelszonen kompatibel, wie es die teilweise Überlappung mit der COMESA-Freihandelszone und das Trade, Development and Cooperation Agreement zwischen Südafrika und der EU beispielhaft belegen (Olivier 2006, S. 62-83). 
stellung von Informationen und die Beratung der Mitgliedstaaten beschränken muss (Oosthuizen 2006, S. 201). Weit schwerer wiegt jedoch, dass sich die Handelsbeziehungen einzelner (Gruppen von) SADC-Staaten zur EU mit Blick auf das Volumen und insbesondere die Zusammensetzung der nationalen Exportwarenkörbe und sensiblen Produktgruppen signifikant unterscheiden (Bilal u. Stevens 2009; Meyn 2010). Daraus ergeben sich nationale Partikularinteressen, die hier exemplarisch angeführt werden:

Südafrika und die BLNS-Staaten exportieren neben Rohstoffen in hohem Maße Agrarprodukte und Nahrungsmittel (insbesondere Fleisch, Früchte, Milchprodukte und Zucker) in die EU, da hier ein komparativer Kostenvorteil besteht. Für Namibia, Mosambik und zu geringem Maße auch Südafrika gilt dies außerdem für Fisch und Meeresfrüchte. Da die Produktpalette der konkurrenzfähigen Exportgüter in die EU bei diesen Ländern einen Schwerpunkt bei ähnlichen Agrarerzeugnissen und Nahrungsmitteln aufweist und die genannten Länder auch innerhalb der Region wirtschaftlich und teilweise auch institutionell - insbesondere die BLNS-Staaten mit Südafrika in der SACU und im Rahmen des Trade, Development and Cooperation Agreement (TDCA) 22 - eng verflochten sind, ergibt sich eine gemeinsame Interessenlage gegenüber der EU. Zum Schutz heimischer Erzeuger und junger Industriezweige wollen die genannten Staaten insbesondere Agrargüter, Textilien, verarbeitete Nahrungsmittel und Produkte der petrochemischen Industrie als sensitive Güter von der Liberalisierung ausnehmen, wobei diese ,gemeinsame Position“ allerdings stark von Südafrikas Wirtschaftsinteressen geprägt ist (McCarthy 2008, S. 118-119; Saurombe 2010, S. 128-129). Die BLNS-Staaten stehen dabei, vor dem Hintergrund ihrer starken wirtschaftlichen Bindung an Südafrika und des bereits bestehenden TDCA, einer relativ raschen Öffnung der eigenen Märkte für mehr als 80 Prozent aller EU-Exporte schrittweise bis 2015 (für Mosambik bis 2023) aufgeschlossen gegenüber (Europäische Kommission 2011).

Für Simbabwe gehören dagegen Tabak, Eisen und Stahl, Baumwolle und Fleischerzeugnisse zu den konkurrenzfähigen Hauptexportgütern in die EU. Mit Blick auf Tabak und Baumwolle gilt dies auch für Malawi, Sambia und in geringerem Maße für Madagaskar. Diese Länder zählen weniger die Agrarerzeugnisse (außer Fleisch) zur Gruppe der sensiblen Güter, sondern neben Baumwolle mit Tabak und Getränken insbesondere auch Produkte der Nahrungs- und Genussmittelindustrie (Meyn u. Kennan 2010, S. 39-40). Harare drängt darüber hinaus auch auf den Schutz seiner (Fuß-)Bekleidungsindustrie, deren Produkte überwiegend regional vermarktet werden (Bilal u. Stevens 2009, 146-161). Gemeinsam mit Südafrika, den BLNS-Staaten und Mosambik in die Verhandlungen mit der EU zu treten, ist für Simbabwe keine Option, da man innerhalb einer solchen Gruppe eine Marginalisierung durch Südafrika befürchtet und darüber hinaus auch eine langsamere Öffnung der eigenen Märkte (für 80 Prozent aller EUExporte schrittweise bis 2022) anstrebt. Mauritius und die Seychellen verfolgen ebenfalls Partikularinteressen und rechnen sich in einer Gruppe ohne Südafrika

22 Das TDCA ist ein Freihandelsabkommen zwischen Südafrika und der EU, dessen Regelwerk aber auch die übrigen Mitglieder der SACU indirekt betrifft; Details dazu im folgenden Kapitel. 
bessere Verhandlungsergebnisse für ein EPA mit der EU aus, insbesondere hinsichtlich der Miteinbeziehung eines Fischereiabkommens (Europäische Kommission 2012a).

Tansania exportiert in die EU neben Edelmetallen insbesondere Kaffee und Tee, aber auch Fisch und Meeresfrüchte, Baumwolle und Tabak. Damit ähnelt die Exportpalette gerade im Hinblick auf das wichtige Exportgut Kaffee auch der seiner Nachbarn Kenia und Uganda, mit denen Tansania auch in der East African Community (EAC) institutionell verflochten ist (Meyn u. Kennan 2010, S. 25-30). Obwohl das Land als LDC mit Blick auf den Marktzugang zur EU im Moment kein EPA abschließen müsste, präferiert es im Einklang mit den ostafrikanischen Nachbarn eine äußerst langsame Marktöffnung (für 82 Prozent aller EU-Exporte schrittweise bis 2033), möchte insbesondere Kaffee und Tee, Gewürze, Fisch und Fleisch als sensitive Güter von der Liberalisierung ausgenommen wissen und optiert auch für ein umfassendes Fischereiabkommen im Rahmen des Partnerschaftsvertrages (Europäische Kommission 2012b). Vor dem Hintergrund seiner Handelsbeziehungen zur EU unterscheidet sich damit Tansanias Position durchaus von denjenigen Südafrikas, der BLNS-Staaten oder Simbabwes.

Es liegt somit nahe, dass eine Liberalisierung und Intensivierung des Außenhandels mit Europa für jene SADC-Staaten mit konkurrenzfähigen Exportprodukten und großer Abhängigkeit von Abnehmern in der EU vorteilhafter ist als eine regionale Zollunion mit geringerem Absatzmarkt und größerem Konkurrenzdruck aufgrund der ähnlichen Exportpaletten der Nachbarstaaten. Da die EPAs vor dem Hintergrund der Aid-for-Trade-Politik (Rat der Europäischen Union 2007) der EU zudem durch Mittel des Europäischen Entwicklungsfonds mit erheblichen Summen finanziell unterfüttert werden, gewinnen sie für die adressierten AKP-Staaten zusätzlich an Attraktivität, da so mögliche Anpassungskosten der bilateralen Handelsliberalisierung kompensiert werden sollen (EU - ACP 2010). Die Fördermittel für die SADC-Region belaufen sich für einen Zeitraum von fünf Jahren auf insgesamt 171 Mio. Euro und jene für die Länder des östlichen und südlichen Afrikas auf insgesamt 302,6 Mio. Euro, wobei ein erheblicher Teil direkt in regionale Programme fließt. ${ }^{23}$ Auch für die LDCs unter den AKPLändern sind die EPAs wegen der inhärenten Entwicklungskomponente daher sehr interessant.

Mit Blick auf die regionale Kooperationsproblematik im Vorfeld der SADCZollunion kann man feststellen, dass die Situation in der SADC im Schatten der starken Wirtschaftsbeziehungen zur EU und unter Berücksichtigung der von ihr offerierten EPAs nun in diesem Politikfeld die Charakteristika eines kooperationsaversen Rambo-Spiels aufweist. Dabei stellen auf regionaler Ebene jene Staaten außerhalb der SADC-EPA Gruppe für die SADC als Organisation die defektierenden Rambos dar, die in der Wirtschaftskooperation mit der EU eine attraktivere und nutzenbringendere Option erkennen als in einer vertieften Marktintegration im Rahmen der geplanten SADC-Zollunion. Durch externe Anreize wird in der SADC eine an sich regionale Problemstruktur somit erheblich verkompliziert, da

23 Information der EU-Kommission:

http://ec.europa.eu/trade/wider-agenda/development/aid-for-trade/programmes/. 10.09.2012. 
auf Partikularinteressen basierende EPAs aufgrund der ihnen inhärenten NordSüd-Freihandelsregime mit dem gemeinsamen Süd-Süd-Integrationsprojekt der geplanten SADC-Zollunion wechselseitig prinzipiell nicht vereinbar sind (Muntschick 2012).

Die EU kann ferner aufgrund der Asymmetrie der wirtschaftlichen Beziehungen zu den AKP-Ländern auf diese in den EPA-Verhandlungen aus einer Position der Stärke heraus Druck ausüben. So fordert die Kommission äußerst nachdrücklich, dass die betroffenen SADC-Staaten die graduelle, reziproke Liberalisierung des Nord-Süd-Handels schnellstmöglich ratifizieren und zumindest im Rahmen von Interim-EPAs institutionalisieren und implementieren (Woolfrey 2009). Käme es bis zum 1. Januar 2014 nicht zum Abschluss der vollständigen EPAs, so die $\mathrm{EU}^{24}$, würde der Zugang zu ihrem Binnenmarkt für die Exporte der SADC-Staaten mit ablehnender Haltung durch den endgültigen Wegfall jeglicher Präferenzbehandlung unweigerlich erheblich erschwert (Europäische Kommission 2011b). Diese Drohungen offenbaren die deutliche Dominanz der EU in den Verhandlungen und stoßen bei den afrikanischen Partnern auf Unverständnis. So erkennt Namibia in der europäischen Verhandlungsführung „Schikane-Taktiken und koloniale Arroganz" 25 und empfindet den von der EU einseitig festgelegten Stichtag als unethisch und unnötig, denn „equal partners do not give each others deadlines." 26 Auch in Südafrika wurde die Verhandlungsstrategie der Europäer als unglücklich, kompromiss- und rücksichtslos wahrgenommen. ${ }^{27}$

Obgleich die Länder des südlichen Afrikas den von der EU ursprünglich angesetzten Stichtag bereits mehrfach und unerwartet erfolgreich hinauszögern konnten (Lorenz 2012), werden sich jene SADC-Staaten mit hohen und volkswirtschaftlich wichtigen europäischen Exportanteilen den wirtschaftlichen Zwängen und dem Druck der EU letztlich kaum entziehen können (Meyn 2010). Gegenwärtig bietet die SADC mit Blick auf die Handelsbeziehungen zur EU ein Bild der inneren Zerrissenheit. Ihre Mitgliedstaaten haben sich in vier EPA-Gruppen zusammengefunden, die gegenwärtig unabhängig voneinander inhaltlich divergierende Handelsregime mit der EU aushandeln:

- Southern African Development Community (SADC)-EPA-Gruppe: Angola, Botsuana, Lesotho, Mosambik, Namibia, Swasiland, Südafrika

- Eastern and Southern Africa (ESA)-EPA-Gruppe: Madagaskar, Malawi, Mauritius, Seychellen, Sambia, Simbabwe

- East African Community (EAC)-EPA-Gruppe: Tansania

- Economic and Monetary Community of Central Africa (CEMAC)-EPAGruppe: DR Kongo

24 Eine aktuelle Entschließung des EU-Parlaments vom 13. September 2012, welcher der Ministerrat noch zustimmen muss, plädiert für eine Verlängerung der Frist auf den 1. Januar 2016. http:// www.europarl.europa.eu/news/en/pressroom/newsletter/2012-W37/19. 18.09.2012.

25 Hage Geingob, Handels- und Industrieminister Namibias. Interview in: Allgemeine Zeitung Windboek, 25.05.2010.

26 Hage Geingob, Handels- und Industrieminister Namibias. Interview in: The Namibian, 09.08.2012.

27 Robert Davies, Handels- und Industrieminister Südafrikas. Interview am Dokumentations- und Kooperationszentrum Südliches Afrika, 07.12.2009.

http://www.sadocc.at/news/2009/2009-167.shtml. 08.09.2012. 
Aus den verschiedenen EPA-Gruppen haben bereits mehrere Länder Interim-EPAs unterzeichnet, die als Vorstufen der vollständigen EPAs den präferenziellen Marktzugang zur EU zunächst provisorisch weiterhin garantieren (Walker 2009). Aus der SADC-EPA-Gruppe haben Botsuana, Lesotho, Swasiland und Mosambik im Juni 2009 diesen Schritt getan (Europäische Kommission 2011a). Folgenschwerer ist jedoch, dass aus der ESA-EPA-Gruppe mit Madagaskar, Mauritius, den Seychellen und Simbabwe im August 2009 vier Mitgliedstaaten der SADC ein von der SADC-Verhandlungsgruppe abweichendes Interim-EPA unterschrieben haben (Europäische Kommission 2012a). Gleiches gilt für Tansania, das unabhängig von seinen SADC-Partnern bereits 2007 im Rahmen der EAC-EPA-Gruppe ein InterimEPA mit der EU abgeschlossen hat (Europäische Kommission 2012b).

Die Unterzeichnung der Interim-EPAs kann als klarer Schritt in Richtung einer vermutlich irreversiblen Institutionalisierung der Nord-Süd-Handelsliberalisierung mit der EU auf Basis von ad hoc koordinierten Ländergruppen, anstatt von bereits etablierten Regionalorganisationen, verstanden werden. Da einige SADC-Staaten gleichzeitig auch Mitglied in anderen, überlappenden Regionalorganisationen wie dem Common Market for Eastern and Southern Africa (COMESA) oder der EAC sind, wirken die EPAs auch dahingehend katalytisch, dass sich die Staaten in der Region gegebenenfalls für eine Regionalorganisation entscheiden müssen (Asche 2008, S. 104).

Angesichts der zentrifugalen Wirkung der EPAs schlägt der SADC in Bezug auf die Zukunft der regionalen Marktintegration nun die Stunde der Wahrheit: Sollten die Mitgliedstaaten der SADC im Rahmen der unterschiedlichen Verhandlungsgruppen tatsächlich separate EPAs nicht nur ratifizieren, sondern auch implementieren, so ist der Weg für ein gemeinsames, einheitliches Abkommen mit der EU verbaut. Da vom Territorium der SADC in diesem Fall mindestens drei voneinander abweichende Handelsregime zur EU etabliert würden, könnte eine alle 15 Mitgliedstaaten umfassende SADC-Zollunion mit gemeinsamem Außenzoll nicht mehr vollendet werden (Walker 2009). Gegenwärtig sieht es so aus, dass dieses Szenario zur Realität wird, denn die EU hat ihre Strategie gegenüber den AKP-Staaten nicht grundlegend geändert und hält damit auch für die in Gruppen zerfallenen SADCLändern die bilaterale EPA-Option offen und gangbar.

\subsection{Gefährdete Marktintegration in der SACU: Droht nach über 100 Jahren ein Auseinanderbrechen der Zollunion?}

Das Kooperationsproblem, das vor mehr als einem Jahrhundert der Schaffung der SACU zugrunde lag, kann heute nicht mehr absolut zweifelsfrei rekonstruiert werden. Retrospektiv könnte es einem Gefangenendilemma geähnelt haben, da die ökonomische Verflechtung zwischen der Südafrikanischen Union und den britischen Protektoraten Bechuanaland, Basutoland und Swasiland damals sehr intensiv war (Blumenfeld 1992, S. 109-118). Die südafrikanische Regierung sah in der SACU dabei anfangs auch ein Instrument zur schrittweisen Inkorporation der kleineren Nachbarn in ihren Wirtschafts- und Hoheitsbereich. Das institutionelle Design der SACU, welches die bestehende Asymmetrie zwischen den BLNS-Staa- 
ten und Südafrika zementiert hat, spiegelt weiterhin die wirtschaftliche Übermacht und den imperialen Einfluss Pretorias von 1910 wider (McCarthy 2003, S. 610-612).

Man könnte nun annehmen, dass die über hundertjährige SACU eine fest etablierte Regionalorganisation ist. Mit dem Ende der Apartheid gewannen jedoch die extra-regionalen ökonomischen Beziehungen der Zollunion zu Europa erheblich an Bedeutung, da das Wirtschaftsembargo gegen Südafrika aufgehoben wurde. Bereits 1994 stellte die EU dem vormals stark isolierten Land ein präferenzielles Handelsabkommen in Aussicht, da sich Südafrika aufgrund der relativen Stärke seiner Volkswirtschaft im Vergleich zu anderen AKP-Staaten nicht für ein Abkommen unter Lomé IV qualifizierte. Das große Interesse der Kaprepublik an einer Revitalisierung und dem Ausbau der Handelsbeziehungen zu seiner wichtigsten Exportdestination führte rasch zu Verhandlungen, die im Jahr 1999 mit der Unterzeichnung des TDCA einen Abschluss fanden (McCarthy 2008, S. 118-119). Das TDCA beinhaltet im Kern die graduelle Schaffung einer bilateralen Freihandelszone zwischen Südafrika und der EU über einen Zeitraum von zwölf Jahren (Rat der Europäischen Union 1999). Die Konditionen fielen für die RSA dabei ausgesprochen vorteilhaft aus: Öffnung des eigenen Marktes für 86 Prozent aller Importe aus der EU, während Brüssel dies in kürzerer Zeit für 95 Prozent aller Importe aus Südafrika garantiert und zusätzlich mit Fördermitteln (980 Mio. Euro für den Zeitraum von 2007-2013) versüßt (Rat der Europäischen Union 2004).

Durch Pretorias SACU-Mitgliedschaft sind auch die BLNS-Länder de facto Teil dieses Handelsabkommens geworden, ohne dass sie jedoch an den Verhandlungen aktiv beteiligt waren (Bertelsmann-Scott 2010, S. 8). Südafrika musste zwar gemäß SACU-Vereinbarung bei Handelsgesprächen zwischen der Zollunion und Dritten die Interessen der BLNS-Staaten offiziell berücksichtigen, doch in der Praxis geschah dies im Fall des TDCA kaum, sodass dessen Regelwerk maßgeblich auf die Belange der RSA zugeschnitten wurde. ${ }^{28}$ Ungeachtet dessen konnte die SACU gegenüber Brüssel als einheitlicher Akteur agieren und ein gemeinsames extra-regionales Handelsregime mit der EU abschließen, sodass das TDCA für die Einheit und Funktionalität der Organisation aus struktureller Sicht zu diesem Zeitpunkt zunächst keine Gefahr darstellte (Frenhoff Larsén 2007; Meyn 2010). Das Abkommen duldet aber keine alternativen, konkurrierenden Freihandelsvereinbarungen anderer SACU-Mitglieder mit der EU, da dies den gemeinsamen AuBenzoll der Organisation untergraben würde (Viner 1950).

Die neue EU-Strategie gegenüber den AKP-Staaten und das Angebot von EPAs an andere Länder im südlichen Afrika drohen nun jedoch die Struktur des der SACU ursprünglich zugrunde liegenden Kooperationsproblems entscheidend zu verändern. Von den SACU-Mitgliedern qualifizieren sich Botsuana, Namibia und Swasiland aufgrund ihres niedrigeren Entwicklungsstandes für die EPA-Verhandlungen. Südafrika darf seit 2007 ebenfalls an den Verhandlungen teilnehmen, obgleich es als Schwellenland nicht unter die Cotonou-Bestimmungen fällt und mit dem TDCA bereits über ein Handelsabkommen mit der EU verfügt (Bilal u.

28 Interview mit Peter Draper (Senior Trade Research Fellow am South African Institute of International Affairs) im Oktober 2010. 
Stevens 2009, S. 20-24). Lesotho genießt als LDC auf Basis der EBA-Initiative ohnehin zollfreien Marktzugang zur EU und müsste daher eigentlich gar kein EPA aushandeln (Europäische Union 2005). Mit Blick auf die Bedeutung des Außenhandels und die wirtschaftliche Zusammenarbeit mit der EU können die EPAs gerade für die weniger entwickelten Länder der SACU eine vielversprechende extra-regionale Kooperationsoption darstellen:

Beispielsweise ist eine der Stützen der Volkswirtschaft Botsuanas der Export von Rindfleisch (nach Diamanten und anderen Mineralien), der kommerzielle Farmer und große Teile der Bevölkerung ernährt. Der Außenhandel mit Europa ist mit Blick auf die Exporte größer als zur eigenen Region, wobei die EU gerade für die nationale Fleischindustrie ${ }^{29}$ als Hauptabnehmer von zentraler Bedeutung ist. Die Regierung in Gaborone erhofft sich von einem EPA neben einer Diversifizierung der eigenen Volkswirtschaft insbesondere verbesserten Zugang zum sicheren, lukrativen und wachsenden europäischen Exportmarkt für heimisches Rindfleisch und Textilprodukte. ${ }^{30}$ Das Interesse an dem Partnerschaftsabkommen gründet aber insbesondere auch auf der Furcht, dass die EU bei Nichtunterzeichnung hohe Importzölle auf Rindfleisch aus Botsuana erheben würde. Dies würde die Konkurrenzfähigkeit des afrikanischen Produkts untergraben und angesichts der großen Abhängigkeit vom europäischen Markt zu einem Kollaps des rindfleischproduzierenden Sektors - und damit zu ländlicher Armut - in Botsuana führen (Grynberg u. Sekolokwane 2009, S. 2).

Namibias extra-regionale Handelsbeziehungen zur EU sind im Vergleich zu anderen SACU-Partnern ebenenfalls sehr intensiv, denn rund 45 Prozent der Gesamtexporte gehen in die EU. Neben Mineralien und Metallen sind es hauptsächlich Lebensmittel, insbesondere Rindfleisch, Fisch und Tafeltrauben, die vornehmlich nach Europa exportiert werden und gut ein Viertel der Gesamtexporte des Landes ausmachen (Grynberg u. Sekolokwane 2009, S. 3). Wie in Botsuana begründet auch in Namibia die Furcht vor einem Verlust des präferenziellen Marktzugangs zur EU für die genannten Waren und den daraus erwachsenden negativen Konsequenzen für die heimischen (Klein-)Farmer den Bedarf an einem EPA. Da Namibia aber im Gegensatz zum Nachbarland insgesamt weniger vom extra-regionalem Handel mit der EU abhängig ist, ist auch seine Position gegenüber Europa reservierter. Die Regierung verlautbart, dass man wohl an einem EPA interessiert sei, es aber nicht gehe, dass ihre „Belange vom Tisch gefegt werden““.31

Die Regierungen von Botsuana, Swaziland und Lesotho befürworten außerdem den europäischen Vorschlag, dass die EPAs neben der Liberalisierung des Güterverkehrs auch den Bereich „Service und Dienstleistungen“ umfassen sollen, da sie sich daraus gesteigerte Wettbewerbsfähigkeit ihrer Märkte und zusätzliche Direktinvestitionen aus der EU erhoffen. Namibia und insbesondere Südafrika lehnen diesen Passus jedoch ab, da sie ihre großen heimischen Servicedienstleister, viele

29 Bedeutendster Akteur ist die parastaatliche Verarbeitungs- und Vertriebsagentur Botswana Meat Commission.

30 Neo Moroka, Handels- und Industrieminister Botsuanas. Interview in: The Botswana Gazette, 17. Juni 2009.

31 Calle Schlettwein, stellvertretender Finanzminister Namibias. Interview in: Allgemeine Zeitung Windhoek, 10.07.2009. 
davon parastaatliche Institutionen, von externer Konkurrenz schützen wollen (Woolfrey 2009, S. 115-116).

Vor dem Hintergrund einer grundsätzlichen Attraktivität der EPAs, die durch die flankierenden Förderprogramme der EU noch aufgewertet werden, könnte sich die Struktur des der SACU ursprünglich zugrunde liegenden Kooperationsproblems in Richtung einer kooperationsaversen Rambo-Situation wandeln. Dabei würden für die Regionalorganisation jene BLNS-Länder zu Rambos, die sich von der extra-regionalen Kooperation mit der EU im Rahmen von individuellen EPAs mehr Gewinne und einen höheren Nutzen versprechen als von der fest institutionalisierten, intra-regionalen Handelsfreiheit und den Zolleinnahmen der SACU. Eine Nutzenkalkulation zugunsten der Kooperation mit externen Akteuren könnte dann zur Folge haben, dass die betroffenen Mitglieder der Zollunion entweder für einen Austritt optieren oder durch eine Implementation von separaten EPAs die Institutionen und die Funktionalität der SACU untergraben.

In der Region gibt es vor dem Hintergrund der EPA-Problematik heftige Spekulationen über die Zukunft der ältesten funktionierenden Zollunion der Welt (Bertelsmann-Scott 2010). So haben Botsuana, Lesotho und Swasiland bereits im Juni 2009 ein Interim-EPA mit der EU unterzeichnet, während Namibia und Südafrika diesen Schritt bislang nicht getan haben (Europäische Kommission 2011). Das Spaltpotenzial der EPAs würde sich für die SACU genau dann entfalten, wenn Botsuana, Lesotho und Swasiland ein umfassendes EPA nicht nur ratifizieren, sondern auch implementieren, während gleichzeitig Namibia und Südafrika dies nicht tun. In diesem Fall würde die Südafrikanische Zollunion mit dem SADC-EPA und dem TDCA zwei unterschiedliche extra-regionale Handelsregime mit der EU aufweisen, was in der Praxis das Ende der SACU bedeuten würde (Viner 1950).

Obgleich die EU mit dem Angebot der attraktiven EPAs innerhalb der SACU erhebliche Zentrifugalkräfte ausgelöst hat, überwiegt bei den BLNS-Staaten der Wert der komplexen intra-regionalen ökonomischen Verflechtung immer noch jenes Potenzial auf Basis ihrer extra-regionalen Beziehungen zur EU (BertelsmannScott 2010). So wird von Namibias Regierung die Ablehnung dieser separaten Abkommen mit der EU explizit damit begründet, dass die regionalen Märkte und das damit verbundene Handelspotenzial für Windhuk letztlich bedeutsamer sind als ein knebelndes, unvorteilhaftes EPA in der aktuellen Form. ${ }^{32}$ Namibias Handels- und Industrieminister betont ferner: "SACU will be finished if the EPA is signed." 33 und damit den Wert der Zollunion für sein Land.

Die SACU ist nicht zuletzt Garant der für die BLNS-Länder so vitalen Zolleinnahmen, sodass diese beim Niedergang der Organisation nicht nur mit enormen finanziellen Einbußen, sondern auch mit wirtschaftlicher und politischer Instabilität rechnen müssten (Draper u. Khumalo 2009). Für Botsuana, dessen Abhängigkeit von den SACU-Zolleinnahmen von allen BLNS-Staaten am geringsten ist, wären die möglichen Kosten von höheren Importzöllen für Rindfleisch bei Verzicht auf ein EPA mit rund 20 Mio. Euro weitaus geringer als die Ausfälle durch

32 Calle Schlettwein, stellvertretender Finanzminister Namibias. Interview in: Namibia Economist, 31.10.2011.

33 Hage Geingob, Handels- und Industrieminister Namibias. Interview in: The Namibian, 9.8.2012. 
ein Auseinanderbrechen der Zollunion in Höhe von mehreren hundert Mio. Euro. Gleiches gilt für Namibia, wo ein Verlust des präferenziellen Marktzugangs zur EU Lebensmittelexporte im Wert von rund 330 Mio. Euro gefährden könnte, ein Zerfall der SACU jedoch den sicheren Wegfall von mehreren hundert Millionen Euro an Einnahmen bedeutet (Grynberg u. Sekolokwane 2009). Maßgeblicher Grund dafür, dass sich Südafrika explizit für den Erhalt der SACU und ein gemeinsames Außenhandelsregime mit der EU einsetzt, ist neben den eigenen wirtschaftlichen Vorteilen insbesondere die Furcht vor einer Destabilisierung der Region, was Absatzmärkte zerstören und hohe wirtschaftliche Kosten verursachen würde (Bertelsmann-Scott 2010, S. 21-22).

Der Ratifizierungsprozess der Interim-EPAs wurde von Botsuana, Lesotho und Swasiland im Jahr 2010 ausgesetzt. Damit ist auch der Abschluss von umfassenden und vollständigen EPAs in der Schwebe, obgleich es weiterhin Verhandlungsrunden zwischen den beiden Seiten gibt (Europäische Kommission 2011). Alle SACU-Länder haben sich auch im selben Jahr darauf geeinigt, dass sie in Zukunft nur noch als gemeinsamer Block in die EPA-Verhandlungen gehen wollen. ${ }^{34} \mathrm{Fer}-$ ner sieht es gegenwärtig so aus, dass es auf Drängen der SACU-Staaten zu einer Harmonisierung des EPA der SADC-Gruppe mit dem bereits bestehendem TDCA kommen wird, da Südafrika die Konditionen seines vorteilhaften Abkommens mit der EU nicht zugunsten eines gemeinsamen SADC-EPA aufgeben will (Julian 2010, S. 14; McCarthy 2010). Die Gefahr, dass sich zwei divergierende Außenhandelsregime zwischen der Südafrikanischen Zollunion und der EU etablieren könnten, wäre damit gebannt. Die EU zeigt sich in dieser Frage zunehmend kompromissbereit, da sie die Bedeutung der SACU für die Region erkannt hat und auch für ein Auseinanderbrechen der Organisation nicht verantwortlich gemacht werden will. ${ }^{35}$

Zusammenfassend lässt sich feststellen, dass aufgrund der dominierenden Stärke der asymmetrischen, intra-regionalen ökonomischen Interdependenz in der SACU die EU als externer Akteur wenig Einfluss auf Dynamik und Zukunft der Marktintegration in dieser Organisation ausüben kann. Obgleich sich die zugrunde liegende Problemstruktur durch das Angebot der EPAs in Richtung einer Rambo-Situation zu verändern droht, wird dieser Zustand wohl kaum erreicht werden. Ein Rambo-Verhalten, das ein Ende der SACU zur Folge hätte, stellt bislang kein Mitgliedsland besser als ein Verbleib in der Zollunion, der den Genuss des gemeinsamen, maßgeblich von Südafrika bereitgestellten Klubgutes (insbesondere in Form von Zolleinnahmen) ermöglicht. Ein Zerfall der SACU ist daher nicht zu erwarten, solange der Zufluss von SACU-Zolleinnahmen generell nicht schwindet, der die BLNS-Staaten begünstigende Verteilungsschlüssel von Südafrika nicht infrage gestellt wird oder die EU den Mehrwert der EPAs für die BLNS-Staaten nicht signifikant erhöht. ${ }^{36}$

34 Dieses Vorgehen steht dann auch im Einklang mit Artikel 32 des SACU-Abkommens, wonach die Mitgliedstaaten ihre Außenhandelsbeziehungen zu Dritten einheitlich regeln müssen.

35 Interview mit Peter Draper (Senior Trade Research Fellow am South African Institute of International Affairs) im Oktober 2010.

36 Interview mit Haile Taye (Senior Research Fellow Macroeconomic Forecasting and Planning am Botswana Institute for Development Policy Analysis) im September 2010. 


\section{Fazit}

Dieser Beitrag hat in einer theoriegeleiteten Analyse die Problematik und den aktuellen Stand der regionalen Marktintegration in der SADC und SACU vorgestellt und erläutert. Es ist deutlich geworden, dass sich Regionalismus im südlichen Afrika vor dem Hintergrund starker und asymmetrischer ökonomischer Interdependenz einer Vielzahl von afrikanischen Ländern zur EU als mächtigem externen Akteur vollzieht. Dieses spezifische Strukturmerkmal beeinträchtigt nicht nur die Situationsstruktur von genuin regionalen Kooperationsproblemen im südlichen Afrika, sondern erlaubt der EU zudem aus einer Position der Stärke heraus Einfluss auf den Status quo und die Zukunft der regionalen Marktintegration in der SADC und SACU auszuüben. Während in der letztgenannten Regionalorganisation die intra-regionale wirtschaftliche Interdependenz der Mitgliedsländer zu Südafrika besonders intensiv und ausschlaggebend ist, gilt dies für die SADC nur in eingeschränktem Maße, da gut die Hälfte ihrer Mitglieder stärkere ökonomische Beziehungen zur EU unterhält. Somit ist die SADC auch prinzipiell einer größeren Einflussnahme durch die EU ausgesetzt.

Die EPAs üben mit den ihnen inhärenten reziproken Freihandelsregimen auf geplante und bereits fest institutionalisierte Marktintegrationsprojekte in der SADC beziehungsweise SACU aus strukturellen Gründen einen negativen Einfluss aus, da sie hohe Anreize zur extra-regionalen Kooperation bieten. Durch die von der EU ausgelobten EPAs ist die Schaffung einer umfassenden und funktionierenden SADC-Zollunion, der alle 15 Mitgliedstaaten angehören, kaum mehr möglich. Obgleich so die regionale Kooperation ,nur ${ }^{6}$ in einem Politikfeld der SADC gestört wird, verwundert dies, da sich die EU ostentativ als Förderer von Regionalismus außerhalb Europas inszeniert. Die Zukunft der SACU scheint dagegen gesichert zu sein, solange sich Südafrika nicht durch externe Anreize oder Druck aus diesem Kooperationsprojekt zurückzieht und die BLNS-Staaten weiterhin von den gemeinsamen Zolleinnahmen mehr profitieren als von unilateralen Handelsabkommen zu Dritten.

Der Artikel leitet die verallgemeinernde Annahme ab, dass Integrationsprozesse in südlichen Regionalorganisationen, deren Konstituenten vornehmlich wirtschaftlich wenig entwickelte und auf regionaler Ebene (ökonomisch) schwächer verflochtene Länder sind, allein aus strukturellen Gründen wesentlich stärker von externen Akteuren negativ beeinflusst werden können als Regionalismus im wirtschaftlich entwickelten und weitaus interdependenteren Norden. Weitere Analysen im Rahmen der vergleichenden Regionalismusforschung könnten die Vermutung erhärten, dass externe Akteure - vielleicht auch die USA oder gar China - auf Regionalorganisationen gerade im Süden störend wirken können, wenn deren Mitglieder zwischen extra-regionalen Kooperationsoptionen und damit unvereinbaren regionalen Integrationsvorhaben wählen müssen. 


\section{Literatur}

Acharya, Amitav, und Alastair Iain Johnston (Hrsg.). 2007. Crafting Cooperation: Regional International Institutions in Comparative Perspective. Cambridge: Cambridge University Press.

Asche, Helmut. 2008. Preserving Africa's economic policy space in trade negotiations. In Negotiating Regions: Economic Partnership Agreements between the European Union and the African Regional Economic Communities, Hrsg. Helmut Asche und Ulf Engel, 79-108. Leipzig: Leipziger Universitätsverlag.

Axelrod, Robert. 1987. Die Evolution der Kooperation. München: R. Oldenbourg.

Axelrod, Robert, und Robert Keohane. 1993. Achieving Cooperation under Anarchy: Strategies and Institutions. In Neorealism and Neoliberalism: The Contemporary Debate, Hrsg. David Baldwin, 85-115. New York: Columbia University Press.

Axline, W. Andrew. 1977. Underdevelopment, dependence and integration: the politics of regionalism in the Third World. International Organization 31: 83-105.

Axline, W. Andrew. 1994. Comparative case studies of regional cooperation among developing countries. In The Political Economy of Regional Cooperation. Comparative Case Studies, Hrsg. W. Andrew Axline, 7-33. London: Pinter Publishers and Associated University Press.

Bach, Daniel. 2003. New Regionalism as an Alias: Regionalisation through Trans-State Networks. In New Regionalism in Africa, Hrsg. Andrew Grant und Fredrik Söderbaum, 21-30. Aldershot: Ashgate.

Bertelsmann-Scott, Talitha. 2010. SACU - One Hundred Not Out: What future for the Customs Union. Johannesburg: South African Institute of International Affairs.

Bilal, Sanoussi, und Christopher Stevens. 2009. The Interim Economic Partnership Agreements between the EU and African States. Contents, challenges and prospects. Maastricht: European Centre for Development Policy Management.

Blumenfeld, Jesmond. 1992. Economic Interdependence in Southern Africa: From Conflict to Cooperation? Kapstadt: Oxford University Press.

Börzel, Tanja, und Thomas Risse. 2009. Diffusing (Inter-) Regionalism. The EU as a Model of Regional Integration. KFG Working Paper Series 7. Berlin: Kolleg Forschergruppe „The Transformative Power of Europe“.

Burns, Tom, und Walter Buckley. 1974. The Prisoners' Dilemma Game as a System of Social Dimension. Journal of Peace Research 11: 221-228.

Draper, Peter, Philip Alves und Mmatlou Kalaba. 2006. South Africa's International Trade Diplomacy: Implications for Regional Integration. Gaborone: Friedrich Ebert Stiftung.

Draper, Peter, und Nkululeko Khumalo. 2009. The Future of the Southern African Customs Union. Trade Negotiations Insights 8: 4-5.

Draper, Peter, Sheila Kiratu und Cézanne Samuel. 2010. The Role of South African FDI in Southern Africa. Bonn: Deutsches Institut für Entwicklungspolitik.

Eckstein, Harry. 1975. Case Study and Theory in Political Science. In Strategies of Inquiry, Hrsg. Fred Greenstein und Nelson Polsby, 79-138. Reading: Addison-Wesley.

Engel, Ulf, und Matthias Middell. 2010. Global cities, new regionalisms, decline and reemergence of the nation state, empire, G 20 or global governance - is there a point of convergence in the debate on changing world orders. In World Orders revisited, Hrsg. Ulf Engel und Matthias Middell, 7-16. Leipzig: Leipziger Universitätsverlag. 
EU - ACP. 2010. Second Revision of the Cotonou Agreement - Agreed Consolidated Text. S. 19-20. http://ec.europa.eu/development/icenter/repository/second_revision_cotonou_ agreement_20100311.pdf. Zugegriffen: 10.09.2012.

European Community - Southern African Region. 2008. Regional Strategy Paper and Regional Indicative Programme 2008-2013.

http://ec.europa.eu/development/icenter/repository/scanned_r7_rsp-2007-2013_en.pdf. Zugegriffen: 19.09.2012.

Europäische Gemeinschaft. 2000. Partnership agreement between the members of the African, Caribbean and Pacific Group of States of the one part, and the European Community and its Member States, of the other part (Cotonou-Abkommen).

http://ec.europa.eu/world/agreements/downloadFile.do?fullText=yes\&treatyTrans $\mathrm{Id}=818$. Zugegriffen: 19.09.2012.

Europäische Kommission. 2002. Recommendation for a Council Decision authorising the commission to negotiate Economic Partnership Agreements with the ACP countries and regions. SEC (2002) 351 final. http://www.eusa.org.za/en/PDFdownload/ Trade\%20\&\%20Economic/EPA_Council_Decision_Recommendation_ACP_ April_2002.pdf. Zugegriffen: 10.09.2012.

Europäische Kommission. 2011a. Fact sheet on the Economic Partnership Agreements. SADC EPA Group. http://trade.ec.europa.eu/doclib/html/142189.htm. Zugegriffen: 02.09.2012.

Europäische Kommission. 2011b. Proposal for a regulation of the European Parliament and of the Council amending Annex I to Council Regulation (EC) No 1528/2007 as regards the exclusion of number of countries from the list of regions or states which have concluded negotiations. http://eur-lex.europa.eu/LexUriServ/LexUriServ.do?uri=C OM:2011:0598:FIN:EN:PDF. Zugegriffen: 10.09.2012.

Europäische Kommission. 2012a. Fact sheet on the Economic Partnership Agreements. Eastern and Southern Africa (ESA). http://trade.ec.europa.eu/doclib/docs/2012/march/ tradoc_149213.pdf. Zugegriffen: 10.09.2012.

Europäische Kommission. 2012b. Fact sheet on the Economic Partnership Agreements. The Eastern African Community (EAC). http://trade.ec.europa.eu/doclib/docs/2009/ january/tradoc_142194.pdf. Zugegriffen: 10.09.2012.

Europäische Union. 2005. Council Regulation (EC) No. 980/2005 of 27 June 2005 applying a scheme of generalised tariff preferences. http://trade.ec.europa.eu/doclib/docs/ 2005/june/tradoc_123910.pdf. Zugegriffen: 10.09.2012.

Frenhoff Larsén, Magdalena. 2007. Trade Negotiations between the EU and South Africa: A Three-Level Game. Journal of Common Market Studies 45: 857-881.

Gehring, Thomas. 1994. Der Beitrag von Institutionen zur Förderung der internationalen Zusammenarbeit. Lehren aus der institutionellen Struktur der Europäischen Gemeinschaft. Zeitschrift für Internationale Beziehungen 1: 211-242.

Gehring, Thomas, und Sebastian Oberthür. 1997. Internationale Regime als Steuerungsinstrumente der Umweltpolitik. In Internationale Umweltregime. Umweltschutz durch Verhandlungen und Verträge, Hrsg. Thomas Gehring und Sebastian Oberthür, 9-26. Opladen: Leske + Budrich.

Goldstein, Andrea. 2004. Regional Integration, FDI and Competitiveness in Southern Africa. Paris: OECD. 
Grobbelaar, Neuma. 2004. Can South African Business Drive Regional Integration on the Continent? South African Journal of International Affairs 11: 91-106.

Grynberg, Roman, und Khutsafalo Sekolokwane. 2009. Signing of the Interim Economic Partnership Agreement. Gaborone: Botswana Institute for Development Policy Analysis.

Haas, Ernst. 1958. The Uniting of Europe: Political, Social, and Economic Forces 1950 1957. Stanford: Stanford University Press.

Hettne, Björn, und Fredrik Söderbaum. 1998. The New Regionalism Approach. Politeia: Journal for Political Science and Public Administration 17: 5-19.

Hirschmann, Albert. 1945. National Power and the Structure of Foreign Trade. Berkeley: University of California Press.

Hurrell, Andrew. 1995. Explaining the resurgence of regionalism in world politics. Review of International Studies 21: 331-358.

Jetschke, Anja, und Tobias Lenz. 2011. Vergleichende Regionalismusforschung und Diffusion: Eine neue Forschungsagenda. Politische Vierteljahresschrift 52: 448-474.

Julian, Melissa. 2010. EPA Update. Trade Negotiations Insights 9: 13-15.

Keck, Alexander, und Roberta Piermartini. 2008. The Impact of Economic Partnership Agreements in Countries of the Southern African Development Community. Journal of African Economies 17: 85-130.

Keohane, Robert. 1984. After Hegemony. Cooperation and Discord in the World Political Economy. Princeton: Princeton University Press.

Keohane, Robert. 1988. International Institutions: Two Approaches. International Studies Quarterly 32: 379-396.

Keohane, Robert, und Joseph Nye. 2001. Power and Interdependence. New York: Longman.

Krapohl, Sebastian, und Simon Fink. 2009. Interdependence vs. Dependence: A Network Analysis of Regional Integration Projects in Africa, America, Asia and Europe. Bamberg Online Papers on Integration Research 3. Bamberg: Universität Bamberg.

Krapohl, Sebastian, und Johannes Muntschick. 2009. Two Logics of Regionalism: The Importance of Interdependence and External Support for Regional Integration in Southern Africa. In Furthering Southern African Integration. Proceedings of the 2008 FOPRISA Annual Conference, Hrsg. Jonathan Kaunda und Francis Zizhou, 3-17. Gaborone: Botswana Institute for Development Policy Analysis.

Lorenz, Ulrike. 2012. Transformations on Whose Terms? Understanding the New EU-ACP Trade Relations from Outside In. KFG Working Paper Series 40. Berlin: Kolleg Forschergruppe „The Transformative Power of Europe“.

Mair, Stefan, und Christian Peters-Berries. 2001. Regionale Integration und Kooperation in Afrika südlich der Sahara. EAC, ECOWAS und SADC im Vergleich. Bonn: Weltforum Verlag.

Mansfield, Edward, und Helen Milner. 1999. The New Wave of Regionalism. International Organization 53: 589-627.

Mattli, Walter. 1999. The Logic of Regional Integration: Europe and Beyond. Cambridge: Cambridge University Press.

McCarthy, Colin. 2003. The Southern African Customs Union in Transition. African Affairs 102: 605-630. 
McCarthy, Colin. 2008. The SADC/SACU interplay in EPA negotiations - A variation on the old theme of integrating unequal economies. In Negotiating Regions: Economic Partnership Agreements between the European Union and the African Regional Economic Communities, Hrsg. Helmut Asche und Ulf Engel, 109-130. Leipzig: Leipzig Universitätsverlag.

Meyn, Mareike. 2010. Die Wirtschaftspartnerschaftsabkommen der Europäischen Union - was war, was ist und was kommen muss. In Afrika und externe Akteure - Partner auf Augenhöhe?, Hrsg. Franziska Stehnken, Antje Daniel, Helmut Asche und Rainer Öhlgeschläger, 75-89. Baden-Baden: Nomos.

Meyn, Mareike, und Jane Kennan. 2010. Economic Partnership Agreements: Comparative Analysis of the Agricultral Provisions. New York: United Nations.

Moravcsik, Andrew. 1998. The Choice for Europe: Social Purpose and State Power from Messina to Maastricht. Ithaca: Cornell University Press.

Muntschick, Johannes. 2012. Theorising Regionalism and External Influence: A Situationstructural Approach. Mainz Papers on International and European Politics 3. Mainz: Chair of International Relations, Johannes Gutenberg-Universität. http://www.politik. uni-mainz.de/cms/Dateien/mpiep02.pdf. Zugegriffen: 10.10.2012.

Nye, Joseph. 1965. Patterns and Catalysts in Regional Integration. International Organization 19: 870-884.

Olivier, Gerrit. 2006. South Africa and the European Union: Self-Interest, Ideology and Altruism. Pretoria: Protea Book House.

Oosthuizen, Gabriël. 2006. The Southern African Development Community. The organisation, its policies and prospects. Midrand: Institute for Global Dialogue.

Pietrangeli, Giulia. 2009. Supporting Regional Integration and Cooperation Worldwide: An Overview of the European Union Approach. In The EU and World Regionalism. The Makability of Regions in the 21st Century, Hrsg. Philippe de Lombaerde und Michael Schulz, 9-43. Farnham: Ashgate.

Powers, Kathy, und Gary Goertz. 2011. The economic-institutional construction of regions: conceptualisation and operationalisation. Review of International Studies 37: 2387-2415.

Rat der Europäischen Union. 1999. Council Decision of 29 July 1999 concerning the provisional application of the Agreement on Trade, Development and Cooperation between the European Community and its Member States, of the one part, and the Republic of South Africa, of the other part. (1999/753/EC).

http://eur-lex.europa.eu/LexUriServ/LexUriServ.do?uri=OJ:L:1999:311:0001:0001:EN :PDF. Zugegriffen: 19.09.2012.

Rat der Europäischen Union. 2004. Council Decision of 26 April 2004 concerning the conclusion of the Trade, Development and Cooperation Agreement between the European Community and its Member States, on the one part, and the Republic of South Africa, on the other part. (2004/441/EC). http://europa.eu/legislation_summaries/development/south_africa/r12201_en.htm. Zugegriffen: 18.09.2012.

Rat der Europäischen Union. 2007. Conclusions of the Council and of the Representatives of the Governments of the Member States meeting within the Council: EU Strategy on Aid for Trade: Enhancing EU support for trade-related needs in developing countries. http://trade.ec.europa.eu/doclib/docs/2008/november/tradoc_141470.pdf. Zugegriffen: 10.09.2012. 
Rohlfing, Ingo. 2009. Bilateralismus und Multilateralismus in den internationalen Beziehungen. Ein polit-ökonomischer Ansatz am Beispiel der Handelskooperation. Zeitschrift für Internationale Beziehungen 16: 75-101.

SACU. 2009. SACU Merchandise Trade Statistics. http://www.sacu.int/publications/trade_ statistics/2011/merchandise_trade_statistics.pdf. Zugegriffen: 04.07.2012.

SACU. 2011. Southern African Customs Union Annual Report 2010/11. http://www.sacu. int/publications/reports/annual/2011/annual2011.pdf. Zugegriffen: 10.09.2012.

SADC. 2004a. Regional Indicative Strategic Development Plan. http://www.sadc.int/attachment/download/file/74. Zugegriffen: 10.07.2012.

SADC. 2004b. Record of the Meeting of SADC Council of Ministers held in Arusha 12-13 March 2004.

SADC. 2006a. Final Communique of the SADC Extraordinary Summit of the Heads of State and Government to consider the Regional, Economic and Political Integration. http://www.info.gov.za/speeches/2006/06102611451001.htm. Zugegriffen: 19.09.2012.

SADC. 2006b. 4th Draft SADC Model Customs Act. http://www.sadc.int/files/1013/2369/ 4831/Final_DRAFT_SADC_Model_CUSTOMS_ACT-doc.doc. Zugegriffen: 04.08.2012.

SADC. 2008. Official SADC Trade, Industry and Investment Review 2007/2008. Hatfield: Trade and Industrial Policy Strategies.

SADC. 2011. Media Briefing on the Outcome of February 2011 SADC Council of Ministers Meeting. http://www.sadc.int/index/browse/page/847. Zugegriffen: 10.09.2012.

Sandrey, Ron. 2011. Intra-African Trade in Southern and Eastern Africa and the Role of South Africa. In Monitoring Regional Integration in Southern Africa. 2010 Yearbook, Hrsg. Anton Bösl, André du Pisani, Gerhard Erasmus, Trudi Hartzenberg und Ron Sandrey, 60-78. Stellenbosch: Trade Law Centre for Southern Africa.

Sandrey, Ron. 2012. Foreign Direct Investment in South Africa. In Monitoring Regional Integration in Southern Africa. Yearbook 2011, Hrsg. Trudi Hartzenberg, Gerhard Erasmus und André du Pisani, 188-213. Stellenbosch: Trade Law Centre for Southern Africa.

Saurombe, Amos. 2010. The role of South Africa in SADC regional integration: the making or braking of the organization. Journal of International Commercial Law and Technology 5: 124-131.

Sbragia, Alberta. 2008. Review Article: Comparative Regionalism: What Might It Be? Journal of Common Market Studies 46: 29-49.

Schirm, Stefan. 2002. Globalization and the New Regionalism: Global Markets, Domestic Politics and Regional Cooperation. Malden: Blackwell Publishers.

Sebenius, James. 1983. Negotiation Arithmetic: Adding and Subtracting Issues and Parties. International Organization 37: 281-316.

Söderbaum, Fredrik. 2007. African Regionalism and EU-African Interregionalism. In European Union and New Regionalism. Regional Actors and Global Governance in a Posthegemonic Era, Hrsg. Mario Telò, 185-202. Aldershot: Ashgate.

Söderbaum, Fredrik, und Patrik Stålgren. 2010. The EU and the Global South. In The European Union and the Global South, Hrsg. Fredrik Söderbaum und Patrik Stålgren, 1-11. Boulder: Lynne Rienner.

Stein, Arthur. 1982. Coordination and Collaboration: Regimes in an Anarchic World. International Organization 36: 300-324.

Viner, Jacob. 1950. The Customs Union Issue. New York: Stevens. 
Vogt, Jörgen. 2007. Die Regionale Integration des südlichen Afrikas. Unter besonderer Betrachtung der Southern African Development Community (SADC). Baden-Baden: Nomos.

Walker, Aurelie. 2009. The EC-SADC EPA: The Moment of Truth for Regional Integration. Trade Negotiations Insights 8: 1, 3.

Warleigh-Lack, Alex, und Luc van Langenhove. 2010. Introduction. Rethinking EU Studies: The Contribution of Comparative Regionalism. Journal of European Integration 32: 541-562.

Woolfrey, Sean. 2009. State of play in the SADC-EU EPA negotiations. In Monitoring Regional Integration in Southern Africa. 2009 Yearbook, Hrsg. Anton Bösl, Gerhard Erasmus, Trudi Hartzenberg und Colin McCarthy, 110-130. Stellenbosch: Tralac.

Young, Oran. 1969. Interdependencies in World Politics. International Journal 24: 726-750. Zangl, Bernhard. 1994. Politik auf zwei Ebenen. Hypothesen zur Bildung internationaler Regime. Zeitschrift für Internationale Beziehungen 1: 279-312.

Zimmerling, Ruth. 1991. Externe Einflüsse auf die Integration von Staaten. Zur politikwissenschaftlichen Theorie regionaler Zusammenschlüsse. Freiburg: Verlag Karl Alber.

Zürn, Michael. 1987. Gerechte internationale Regime. Bedingungen und Restriktionen der Entstehung nicht-hegemonialer internationaler Regime untersucht am Beispiel der Weltkommunikationsordnung. Frankfurt a. M.: Haag Herchen.

Zürn, Michael. 1992. Interessen und Institutionen in der Internationalen Politik: Grundlegung und Anwendung des situationsstrukturellen Ansatzes. Opladen: Leske + Budrich.

Zürn, Michael. 1993. Problematic Social Situations and International Institutions: On the Use of Game Theory in International Politics. In International Relations and PanEurope: Theoretical Approaches and Empirical Findings, Hrsg. Frank Pfetsch, 63-84. Münster: LIT Verlag.

\section{Autorenangaben:}

Dipl. Pol. Johannes Muntschick,

Johannes Gutenberg-Universität Mainz, Jakob-Welder-Weg 12, 55099 Mainz, muntschick@uni-mainz.de 